\title{
Effect of paternal age on treatment outcomes in couples undergoing assisted reproductive technology for non-male factor infertility: a retrospective analysis of 809 cycles
}

Treasa Joseph ${ }^{1}$, Reka Karuppusami ${ }^{2}$, Muthukumar Karthikeyan ${ }^{1}$, Aleyamma T. Kunjummen ${ }^{1}$ and Mohan S. Kamath ${ }^{1 *}$

\begin{abstract}
Background: Worldwide, there is an increase in uptake of assisted reproductive technology (ART) treatment. The impact of paternal age on ART outcomes is unclear. In view of the conflicting results reported by studies evaluating effect of paternal age on ART outcomes, we planned a study to investigate the impact of advanced paternal age in couples undergoing ART for non-male factor infertility. We conducted a retrospective cohort study at a universitylevel teaching hospital in South India. All couples who underwent ART for non-male factor infertility were included. The couples were divided into two groups based on the age of the male partner. Group I included couples with male partner's age less than 40 years, taken as reference group. Group II included couples with male partners age more than or equal to 40 years. The primary outcome was live birth rate. Secondary outcomes included clinical pregnancy, miscarriage, fertilization, embryo development, and blastulation rates.
\end{abstract}

Results: A total of 809 cycles were included for the study. Following exclusion of 39 cycles, 770 cycles were analyzed for outcomes. Group I comprised of 556 (72\%) cycles and group II comprised of 214 (28\%) cycles. There was no significant difference in live birth rate per embryo transfer between groups I and II (31.8\% vs. 29.4\%; odds ratio, OR, 0.89; $95 \% \mathrm{Cl} 0.63$ to 1.26). After adjustment for potential confounders, the live birth rate did not differ significantly (adjusted odds ratio, aOR, 1.10; $95 \% \mathrm{Cl} 0.74$ to 1.65$)$. The clinical pregnancy (39.4\% vs. 36\%; aOR 1.06; $95 \% \mathrm{Cl} 0.72$ to 1.56) and the miscarriage rates ( $18.3 \%$ vs. $15.6 \%$; aOR $0.73 ; 95 \% \mathrm{Cl} 0.32$ to 1.66$)$ were also similar between the two groups. There was significant decrease in the blastulation rate (36.8\% vs. $32.1 \% ; P$ 0.002) in the advanced paternal age group as compared to the reference group.

Conclusion: The current study suggests that in couples undergoing ART for non-male factor, there is no detrimental effect of increasing paternal age on treatment outcomes.

Keywords: Paternal age, Assisted reproductive technology, Live birth

\footnotetext{
* Correspondence: dockamz@gmail.com

'Department of Reproductive Medicine, Christian Medical College, Vellore

632004, India

Full list of author information is available at the end of the article
}

\section{Springer Open}

(c) The Author(s). 2020 Open Access This article is licensed under a Creative Commons Attribution 4.0 International License, which permits use, sharing, adaptation, distribution and reproduction in any medium or format, as long as you give appropriate credit to the original author(s) and the source, provide a link to the Creative Commons licence, and indicate if changes were made. The images or other third party material in this article are included in the article's Creative Commons licence, unless indicated otherwise in a credit line to the material. If material is not included in the article's Creative Commons licence and your intended use is not permitted by statutory regulation or exceeds the permitted use, you will need to obtain permission directly from the copyright holder. To view a copy of this licence, visit http://creativecommons.org/licenses/by/4.0/. 


\section{Background}

Inability to conceive after 1 year of unprotected sexual intercourse is known as infertility, and one in six couples in the reproductive age group is diagnosed as infertile [1]. Worldwide, there is an increase in uptake of assisted reproductive technology (ART) treatment [1]. For many indications such as bilateral tubal damage, severe endometriosis, and male factor, ART remains the most effective treatment option [2]. Due to sociodemographic changes across the world, ART is being increasingly offered to couples aged 40 years and beyond [3].

Maternal age remains one of the important prognostic factors for ART success. Studies have reported a steep decline in live birth rates with ART after the age of 40 years [4-6]. This decline in live birth is mainly attributed to age-related decline in oocyte quality and quantity, increase in aneuploidy, reduced fertilization, and lower implantation rates $[7,8]$.

The impact of paternal age on fertility treatment such as intrauterine insemination (IUI) and ART outcomes is unclear. Studies evaluating the effect of paternal age on IUI cycles reported no association of paternal age with pregnancy outcomes $[9,10]$. An earlier study reported a significant negative influence of increasing paternal age on pregnancy rate following ART [5], while other studies did not find any association of paternal age on ART outcomes [11-13]. It is suggested that advanced paternal age affects fertility partly due to the changes in semen parameters, such as sperm motility and morphology [14], but mostly due to genetic alterations and de novo mutations in the male germ cells [15].

Although many studies have reported effects of paternal age in male factor infertility $[5,8]$, there is a paucity of studies evaluating the effect of paternal age in nonmale factor infertility [16]. Hence, we planned a study to investigate the impact of advanced paternal age in couples undergoing ART for non-male factor infertility.

\section{Methods}

We conducted a retrospective cohort study. The study was carried out in the Department of Reproductive Medicine of a university-level teaching hospital in South India. All couples who underwent ART for non-male factor infertility and resulted in a fresh or frozen embryo transfer between July 2010 and June 2018 were included in the study. The type of ART treatment included either intracytoplasmic sperm injection (ICSI) or a combination of in vitro fertilization (IVF) and ICSI. As this was a retrospective study, ethics committee approval was not required.

We included all couples who underwent ART, and the male partner had a normal diagnostic semen analysis. Semen volume was determined using wide-bore volumetric pipette, and motility was assessed by wet slide preparation. Concentration was assessed using Neubauer's counting chamber, and morphology was assessed using prestained slides and graded by Kruger's criteria. The analysis of semen parameters was based on lower reference ranges as defined by the WHO 2010 criteria [17]. We excluded the following couples: (i) female partner's age more than 40 years, (ii) poor response (less than or equal to 4 oocytes obtained on oocyte retrieval), and (iii) cycles where cryopreserved semen sample was used. We included only a single ART cycle for each couple for analysis of outcomes.

We divided the cases into two groups based on the age of the male partner. Group I included couples where the male partner's age was less than 40 years and was chosen to be the reference group. Group II included couples with male partner's age more than or equal to 40 years.

We used conventional long GnRH agonist, GnRH antagonist, or ultralong protocols depending on indication for ART. Controlled ovarian hyperstimulation was done using recombinant FSH (between 100 and $300 \mathrm{IU})$, and follicular monitoring was carried out by serial transvaginal ultrasound. An hCG trigger of 5000 IU was administered when at least 3 follicles $\geq$ $17 \mathrm{~mm}$ were seen on ultrasound. Oocyte retrieval was done after $35 \mathrm{~h}$, after the trigger. Between one and three embryos were transferred either at cleavage (day 2 or 3) or blastocyst stage (day 5). Luteal support was given with intravaginal micronized progesterone, 400 mg twice daily, and intramuscular progesterone (100 mg) was administered twice weekly. Pregnancy was confirmed by checking serum beta hCG levels on day 18 after oocyte retrieval.

In cycles where an elective cryopreservation of embryos was done, a frozen embryo transfer was planned after artificial preparation of the endometrium was done using escalating doses of oral estrogen valerate and intravaginal progesterone. The transfer was planned according to the day of embryo cryopreservation. Luteal support was the same as that given for fresh cycles. The serum beta hCG was checked on the 18th day of starting progesterone.

Information regarding clinical and laboratory variables such as age, indication, oocyte numbers, embryo quality, number of embryos transferred, type of transfer (fresh vs. frozen), and day of transfer (cleavage vs. blastocyst) were obtained from the departmental ART database. The pregnancy outcomes were collected from the women through e-mails and telephone contacts. Collected data were entered in SPSS, and data were analyzed using STATA, version 21.0 (Statacorp).

The primary outcome was live birth per embryo transfer. Live birth was defined as delivery after 24 completed weeks of gestation. Secondary outcomes included clinical 
pregnancy rate, miscarriage rate, fertilization rate, embryo development rate, and blastulation rate.

Clinical pregnancy was defined as presence of a gestational sac on ultrasound between 6 and 8 weeks of gestation. Miscarriage rate was defined as the number of spontaneous losses of the fetus or the absence of cardiac activity prior to 24 weeks divided by the number of clinical pregnancies. Fertilization rate was defined as the total number of fertilised oocytes divided by the total number of inseminated or injected oocytes. Embryo development rate was defined as the total number of embryos which have undergone cleavage divided by the total number of fertilised oocytes. Blastulation rate was defined as total number of blastocysts divided by the number of cleaved embryos.

Data were reported as mean (SD) for continuous variables and frequency (percentages) for categorical variables. $t$ test was used to compare both groups. To find out the association between two categorical data, the chi-square test was used. Fertilization rate, embryo development rate, and blastulation rate were compared between the age groups using proportion test. Dichotomous outcomes (live birth, miscarriage, and clinical pregnancy) were analyzed by simple logistic regression. Multiple logistic regression models were constructed to control for potential confounders such as female age, female body mass index, indication for ART, day of transfer (cleavage vs. blastocyst), and type of transfer (fresh vs. frozen) and assess the association between paternal age and outcomes. The effect is reported as odds ratio (OR) with 95\% confidence interval (CI). Data were analyzed using SPSS, version 21.0 (Armonk, NY: IBM Corp).

\section{Results}

A total of 2849 ART cycles were performed during the study period. After excluding 2040 cycles for reasons as

Female age $>40$ years $(n=81)$

Male factor $(\mathrm{n}=1030)$

Oocytes retrieved $\leq 4(n=589)$

Surgically retrieved or cryopreserved

semen sample $(n=340)$

Total number of ART cycles satisfying

inclusion criteria $(n=809)$

Did not complete cycle $(\mathrm{n}=13)$

Lost to follow up $(n=26)$

Total number of ART cycles included for analysis $(n=770)$

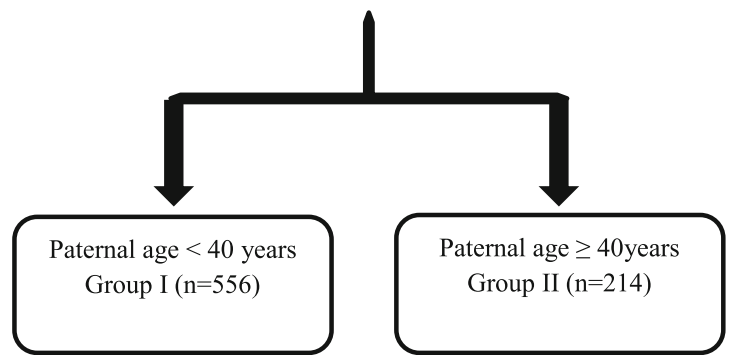

Fig. 1 Algorithm for recruitment of cases 
Table 1 Baseline characteristics of group I (male partner age < 40 years) and group II (male partner $\geq$ years)

\begin{tabular}{|c|c|c|c|}
\hline & Group I $(<40$ years) $(\boldsymbol{n}=556)(\%)$ (reference group) & Group II ( $\geq 40$ years) $(\boldsymbol{n}=214)(\%)$ & $\boldsymbol{P}$ value \\
\hline Mean female age (years)* & $31.08(3.8)$ & $35.14(3.4)$ & $0.01^{\#}$ \\
\hline Mean female BMI* & $25.04(3.9)$ & $26.62(4.7)$ & $0.01^{\#}$ \\
\hline Previous parity & $31 / 556(5.6)$ & $32 / 214(15.0)$ & $0.02^{\#}$ \\
\hline H/o smoking & $85 / 556(15.3)$ & $29 / 214(13.5)$ & 0.67 \\
\hline Primary infertility & $365 / 556(65.6)$ & $119 / 214(55.6)$ & $0.01^{\#}$ \\
\hline Indication-tubal factor & $141 / 556(25.3)$ & $50 / 214(23.3)$ & 0.05 \\
\hline No. of oocytes retrieved ${ }^{\dagger}$ & $9(6,14)$ & $8(5,13)$ & $0.01^{\#}$ \\
\hline ICSI cycles & $325 / 556(58.4)$ & $143 / 214(66.8)$ & 0.06 \\
\hline \multicolumn{4}{|l|}{ Day of transfer } \\
\hline Cleavage stage & $411 / 556(73.9)$ & $167 / 214(78.1)$ & \multirow[t]{2}{*}{0.45} \\
\hline Blastocyst stage & $145 / 556(26.1)$ & $47 / 214(21.9)$ & \\
\hline No. of embryos transferred ${ }^{\dagger}$ & $2(2,3)$ & $2(2,3)$ & 0.43 \\
\hline \multicolumn{4}{|l|}{ Type of transfer } \\
\hline Fresh transfer & $417 / 556(75)$ & $183 / 214$ (85.5) & \multirow[t]{2}{*}{$0.02^{\#}$} \\
\hline Frozen transfer & 139/556 (25) & $31 / 214(14.5)$ & \\
\hline
\end{tabular}

$B M I$ body mass index, ICSI intracytoplasmic sperm injection

* Mean (standard deviation)

tMedian (interquartile range)

"Statistically significant

shown in Fig. 1, a total of 809 ART cycles were included in the study. Additionally, 39 cycles were excluded for not completing the treatment cycle $(n=13)$ or were lost to follow up $(n=26)$. Finally, 770 ART cycles were analyzed for the planned outcomes (Fig. 1).

A total of 556/770 (72\%) cycles were included in group I (male partner age $<40$ years) and 214/770 (28\%) cycles were included in group II (male partner age $\geq 40$ years). The baseline parameters were compared, and significant difference was observed for the following parameters: female age, mean female body mass index (BMI), previous parity, type of infertility, number of oocytes retrieved, and fresh or frozen transfer (Table 1).

There was no significant difference in live birth rate per embryo transfer between groups I and II (31.8\% vs. $29.4 \%$; OR $0.89,95 \%$ CI 0.63 to $1.26 ; P 0.52$ ). After the adjustment for potential confounders, the live birth rate did not differ significantly (aOR 1.10; 95\% CI 0.74 to $1.65 ; P 0.64)$. There were no significant differences in clinical pregnancy $(39.4 \%$ vs. $36 \%$;
aOR 1.06; $95 \%$ CI 0.72 to $1.56 ; P$ 0.75) and the miscarriage rates $(18.3 \%$ vs. $15.6 \%$; aOR $0.73 ; 95 \%$ CI 0.32 to $1.66 ; P$ 0.45) observed between the two groups (Table 2).

There were no significant differences in fertilization (76.8\% vs. $75.3 \%$; OR 0.92 ; $95 \%$ CI 0.81 to $1.05 ; P 0.20$ ) and the embryo development rates $(96.2 \%$ vs. $95.6 \%$; OR 0.86 ; $95 \%$ CI 0.63 to1.18; $P$ 0.33), but there was a significant decrease in the blastulation rate $(36.8 \%$ vs. $32.1 \%$; OR $0.81 ; 95 \%$ CI 0.71 to $0.93 ; P 0.002$ ) in the advanced paternal age group as compared to the reference group (Table 3).

The semen volume was found to be significantly lower $(2.7 \pm 1.31$ vs. $2.6 \pm 1.36 \mathrm{ml} ; P 0.02)$ in the advanced age group while no significant difference was seen in the sperm concentration (61, interquartile range, IQR 33 to 101 vs. 70 , IQR 38 to101 million per ml; $P$ 0.06), motility (53\%, IQR 44 to $57 \%$ vs. $52 \%$, IQR 42 to $56 \%$; $P$ 0.21), and morphology (5\%, IQR 3 to $5 \%$ vs. $5 \%$, IQR 3 to $5 \%$; $P$ 0.51) between the two groups (Table 4).

Table 2 Comparison of clinical outcomes between group I (male partner age $<40$ years) and group II (male partner $\geq$ years)

\begin{tabular}{lllll}
\hline & Group I (<40 years) (reference group) & Group II ( $\geq 40$ years) & OR (95\% Cl) & aOR (95\% CI) \\
\hline Live birth rate* $^{*}$ & $177 / 556(31.8 \%)$ & $63 / 214(29.4 \%)$ & $0.89(0.63$ to 1.26$)$ & $1.10(0.74$ to 1.65$)$ \\
Clinical pregnancy rate $^{*}$ & $219 / 556(39.4 \%)$ & $77 / 214(36.0 \%)$ & $0.86(0.62$ to 1.20$)$ & $1.06(0.72$ to 1.56$)$ \\
Miscarriage rate $^{\dagger}$ & $40 / 219(18.3 \%)$ & $12 / 77(15.6 \%)$ & $0.83(0.41$ to 1.67$)$ & $0.73(0.32$ to 1.66$)$ \\
\hline
\end{tabular}

aOR adjusted odds ratio-adjusted for female age, female body mass index, indication for ART, day of transfer (cleavage vs. blastocyst), and type of transfer (fresh vs. frozen), $A R T$ assisted reproductive technology, $C l$ confidence interval, $O R$ odds ratio

*Calculated per embryo transfer

tCalculated per clinical pregnancy 
Table 3 Comparison of laboratory outcomes between group I (male partner age $<40$ years) and group II (male partner $\geq$ years)

\begin{tabular}{llll}
\hline & Group I (<40 years) (reference group) & Group II ( $\geq 40$ years) & OR (95\% Cl) \\
\hline Fertilization rate & $3874 / 5042(76.8 \%)$ & $1295 / 1719(75.3 \%)$ & $0.92(0.81$ to 1.05$)$ \\
Embryo development rate & $3726 / 3874(96.2 \%)$ & $1238 / 1295(95.6 \%)$ & $0.86(0.63$ to 1.18$)$ \\
Blastulation rate & $1371 / 3726(36.8 \%)$ & $398 / 1238(32.1 \%)$ & $0.81(0.71 \text { to } 0.93)^{\#}$ \\
\hline
\end{tabular}

$O R$ odds ratio, $\mathrm{Cl}$ confidence interval

\#Statistically significant

We carried out a subgroup analysis to evaluate the effect of advanced paternal age in young women $(\leq 35$ years). There was no significant difference in live birth (32.1\% vs. $35.2 \%$; OR 1.15 ; $95 \%$ CI 0.74 to $1.78 ; P 0.53)$, clinical pregnancy $(39.4 \%$ vs. $43.8 \%$; OR $1.14 ; 95 \%$ CI 0.75 to $1.74 ; P 0.53)$, and miscarriage rates $(17.5 \%$ vs. 13.0\%; OR 0.71; $95 \%$ CI 0.28 to $1.80 ; P 0.47$ ) between the two groups. The results were similar after adjustment of various confounding factors (Table 5).

\section{Discussion}

The current study showed no difference in live birth rates following ART in couples with advanced paternal age ( $\geq 40$ years) compared to those couples in whom the paternal age was $<40$ years. There was no significant difference in clinical pregnancy and miscarriage rates in both the groups. While the fertilization rate and the embryo development rate were similar in both the groups, the blastulation rate was significantly lower in the advanced paternal age group.

Klonoff Cohen et al. in a retrospective study showed lower pregnancy rates with advanced age $>35$ years. The investigators reported an $11 \%$ increase in odds of not achieving a pregnancy with every one year increase in paternal age [6]. The differences in the paternal age groups compared, inclusion of previous failed cycles, and inclusion of cycles with different methods of fertilization (GIFT and IVF) may be the reasons for contradictory findings compared to the current study. Conversely, another retrospective study including 2627 ICSI cycles with men aged between 22 and 78 years reported increased negative influence of paternal age on the number of high-quality embryos but no impact on treatment outcomes such as clinical pregnancy, miscarriage, and live birth rates [18]. Luna et al. in an analysis of donor oocyte cycles showed significant decrease in blastulation when paternal age $>40$ years [12]. The decrease in blastulation may be explained by the activation of a compromised paternal genome due to advanced age during compaction of the embryo. The results of another retrospective study of 1023 oocyte donor cycles suggested a significant decrease in live birth rate and significant increase in miscarriage rate in couples with male partners' age $>50$ years [5]. However, the quality of evidence presented by the above study was suggested to be medium in another systematic review due to its observational nature and potential for bias [19]. This review which included an analysis of 12,538 oocyte donation cycles further concluded that there was no statistical correlation between advanced paternal age and live birth, pregnancy, miscarriage, fertilization, or embryo development. A systematic review of 10 retrospective studies showed no significant negative correlation between advanced paternal age and live birth, clinical pregnancy, miscarriage, and fertilization [20]. However, the same review suggested a significant decrease in blastulation with increasing paternal age.

A retrospective study on 4887 oocyte donation cycles by Begeuria et al. reported significant decrease in semen volume and sperm motility and a significant increase in sperm concentration with advanced paternal age [21]. The reason for this contradictory finding could be the use of cryopreserved semen samples and semen samples with abnormal parameters.

In the current study, comparison of semen parameters showed significant decrease in semen volume and no significant difference in sperm concentration, motility, and morphology in couples with paternal age $>40$ years. A recent retrospective study including 70 men with normal semen parameters and normal DNA fragmentation

Table 4 Comparison of semen parameters between group I (male partner age $<40$ years) and group II (male partner $\geq$ years)

\begin{tabular}{llll}
\hline & Group I (<40 years) (reference group) $(\boldsymbol{n}=556)$ & Group II ( $\geq 40$ years) $(\boldsymbol{n}=214)$ & $\begin{array}{l}\boldsymbol{P} \\
\text { value }\end{array}$ \\
\hline Volume, m|* & Median (IQR) & $2.6(1.36)$ & $0.02^{\#}$ \\
Concentration, million/ml & $2.7(1.31)$ & $70(38$ to 101) & 0.06 \\
Motility, \% & $61(33$ to 101$)$ & $52(42$ to 56$)$ & 0.21 \\
Morphology, \% & $53(44$ to 57$)$ & 5 (3 to 5) & 0.51 \\
\hline
\end{tabular}

IQR interquartile range, $\mathrm{m} /$ milliliter

*Mean (standard deviation)

"Statistically significant 
Table 5 Subgroup analysis: comparison of clinical outcomes between group I (male partner age < 40 years) and group II (male partner $\geq$ years) in women $\leq 35$ years

\begin{tabular}{lllll}
\hline & Group I (<40 years) (reference group) $(\boldsymbol{n}=493)$ & Group II $(\geq 40$ years $)(\boldsymbol{n}=108)$ & OR $(95 \% \mathrm{Cl})$ & aOR $(95 \% \mathrm{Cl})$ \\
\hline Live birth rate $^{*}$ & $158 / 493$ & $38 / 108$ & $1.15(0.74,1.78)$ & $1.18(0.75,1.86)$ \\
& $32.1 \%$ & $35.2 \%$ & $1.14(0.75,1.74)$ & $1.13(0.73,1.76)$ \\
Clinical pregnancy rate $^{*}$ & $194 / 493$ & $46 / 108$ & \\
Miscarriage rate $^{\dagger}$ & $39.4 \%$ & $43.8 \%$ & $0.71(0.28,1.80)$ & $0.63(0.23,1.70)$ \\
& $34 / 194$ & $6 / 46$ & $13.0 \%$ & \\
\hline
\end{tabular}

aOR adjusted odds ratio-female body mass index, indication for ART, day of transfer (cleavage vs. blastocyst), and type of transfer (fresh vs. frozen), ART assisted reproductive technology, $\mathrm{Cl}$ confidence interval, $O R$ odds ratio

*Calculated per embryo transfer

† Calculated per clinical pregnancy

index showed no significant difference in semen volume, motility, and morphology between men $\geq 45$ years compared to those $<45$ years [22]. However, Dain et al. in his systematic review suggested a significant decrease in volume with advancing paternal age [20]. This decrease can be explained by reduced androgen-stimulated fluid production from the seminal vesicles and prostate [20]. The same review did not find a significant difference in concentration, motility, and morphology in the advanced paternal age group.

The current study is one of the few studies evaluating the effect of advanced paternal age in couples undergoing ART for non-male factor infertility. Among the strengths of the current study are the inclusion of large number of ART cycles and including live birth rate as an outcome. We excluded potential confounders such as advanced maternal age, poor responders, and male factor infertility. While we have also adjusted for known potential confounders, it is possible that residual confounders may have influenced the results. One of the limitations of the study is the retrospective design. Another factor was that the lack of objective assessment of DNA damage.

\section{Conclusion}

In couples undergoing ART for non-male factor, the current study suggests no detrimental effect of increasing paternal age on treatment outcomes. However, a larger study with inclusion of larger proportion of men with advanced age ( $>50$ years) is needed to validate the current study findings.

\section{Abbreviations}

ART: Assisted reproductive technology; IVF: In vitro fertilization; ICSI: Intracytoplasmic sperm injection; GnRH: Gonadotrophin releasing hormone; IU: International units; hCG: Human chorionic gonadotrophin; GIFT: Gamete intrafallopian transfer

\section{Acknowledgements}

We would like to acknowledge all the couples who were included in the study.

\section{Authors' contributions}

TJ conceptualized and planned the design of the study, acquired the data, and drafted the manuscript. RK analyzed the data and interpreted the results. MK and ATK revised the manuscript for important intellectual content. MSK interpreted the results and refined the manuscript critically. All authors read and approved the final manuscript.

Funding

No funding was taken for the study.

\section{Availability of data and materials}

The datasets used and/or analyzed during the current study are available from the corresponding author on reasonable request.

Ethics approval and consent to participate

As this was a retrospective study, ethics committee approval was not required.

\section{Consent for publication}

Not applicable.

\section{Competing interests}

The authors declare that they have no competing interests.

\section{Author details}

'Department of Reproductive Medicine, Christian Medical College, Vellore 632004, India. ${ }^{2}$ Department of Biostatistics, Christian Medical College, Vellore 695002, India.

Received: 13 January 2020 Accepted: 4 May 2020

Published online: 19 May 2020

\section{References}

1. Adamson GD, de Mouzon J, Chambers GM, Zegers-Hochschild F, Mansour $\mathrm{R}$, Ishihara $\mathrm{O}$ et al (2018) International Committee for Monitoring Assisted Reproductive Technology: world report on assisted reproductive technology, 2011. Fertil Steril 110(6):1067-1080

2. Huang L, Tan J, Hitkari J, Dahan M (2015) Should IVF be used as first-line treatment or as a last resort? A debate presented at the 2013 Canadian Fertility and Andrology Society meeting. Reproductive BioMedicine Online 30(2):128-136

3. A.P. Ferraretti, K. Nygren, A. Nyboe Andersen, J. de Mouzon, M. Kupka, C. Calhaz-Jorge,etal, Trends over 15 years in ART in Europe: an analysis of 6 million cycles, Human Reproduction Open, Volume 2017, Issue 2, 2017.

4. Ventura SJ, Mosher WD, Curtin SC, AbmaJC, Henshaw S. Trends in pregnancies and pregnancy rates by outcome: estimates for the United States 1976-96. Vital Health Stat 21 2000; 56:1-47.

5. Frattarelli JL, Miller KA, Miller BT, Elkind-Hirsch K, Scott RT Jr (2008) Male agenegatively impacts embryo development and reproductive outcome in donor oocyte assisted reproductive technology cycles. FertilSteril 90:97-103

6. Klonoff-Cohen $\mathrm{H}$, Natarajan $\mathrm{L}$ (2004) The effect of advancing paternal age on pregnancy and live birth rates in couples undergoing in vitro fertilization or gamete intrafallopian transfer. American Journal of Obstetrics and Gynecology. 191(2):507-514 
7. Munne S, Alikani M, Tomkin G, Grifo J, Cohen J. Embryo morphology,developmental rates, and maternal age are correlated with chromosome abnormalities. Fertil Steril 1995; 64:382-391.

8. Pantos K, Athanasiou V, Stefanidis K, Stavrou D, Vaxevanoglou T, Chronopoulou M (1999) Influence of advanced age on the blastocyst development rate and pregnancy rate in assistedreproductive technology. FertilSteril 71:1144-1146

9. Tatsumi T, Ishida E, Tatsumi K, Okada Y, Saito T, Kubota T et al (2018) Advanced paternal age alone does not adversely affect pregnancy or livebirth rates or sperm parameters following intrauterine insemination. Reproductive Medicine and Biology. 17(4):459-465

10. Ghuman N, Mair E, Pearce K, Choudhary M (2016) Does age of the sperm donor influence live birth outcome in assisted reproduction? Human Reproduction. 31(3):582-590

11. Gallardo E, Simon C, Levy M, GuanesPP RJ, Pellicer A (1996) Effect of age onsperm fertility potential: oocyte donation as a model. FertilSteril 66:260-264

12. Luna M, Finkler E, Barritt J, Bar-Chama N, Sandler B, CoppermanAB GL (2009) Paternal age and assisted reproductive technology outcomein ovum recipients. FertilSteril 92:1772-1775

13. Aboulghar M, Mansour R, Al-Inany H, Abou-Setta A, Aboulghar M, Mourad L et al (2007) Paternal age and outcome of intracytoplasmic sperm injection. Reproductive BioMedicine Online 14(5):588-592

14. Stone BA, Alex A, Werlin LB, Marrs RP (2013) Age thresholds for changes in semen parameters in men. FertilSteril 100:952-958

15. Zhu JL, Madsen KM, Vestergaard M, OlesenAV BO, Olsen J (2005) Paternal age and congenital malformations. Hum Reproduction 20:3173-3177

16. Kaarouch I, Bouamoud N, Madkour A, Louanjli N, Saadani B, Assou S et al (2018) Paternal age: Negative impact on sperm genome decays and IVF outcomes after 40 years. Molecular Reproduction and Development. 85(3): 271-280

17. Cooper T, Noonan E, von Eckardstein S, Auger J, Baker H, Behre $\mathrm{H}$ et al (2009) World Health Organization reference values for human semen characteristics*. Human Reproduction Update. 16(3):231-245

18. Wu Y, Kang X, Zheng H, Liu H, Huang Q, Liu J (2016) Effect of paternal age on reproductive outcomes of intracytoplasmic sperm injection. PLOS ONE. 11(2):e0149867

19. Sagi-Dain L, Sagi S, Dirnfeld M. Effect of paternal age on reproductive outcomes in oocyte donation model: a systematic review. Fertility and Sterility. 2015; 104(4):857-865.e1.

20. Dain L, Auslander R, Dirnfeld M (2011) The effect of paternal age on assisted reproduction outcome. Fertility and Sterility. 95(1):1-8

21. Begueria R, Garcia D, Obradors A, Poisot F, Vassena R, Vernaeve V (2014) Paternal age and assisted reproductive outcomes in ICSI donor oocytes: is there an effect of older fathers? Human Reproduction. 29(10):2114-2122

22. Darbandi S, Darbandi M, Khorram Khorshid H, Sadeghi M, Heidari M, Cheshmi G et al (2019) The effect of paternal age on semen quality and fertilization outcome in men with normal sperm DNA compaction, reactive oxygen species, and total antioxidant capacity levels. Türk Üroloji Dergisi/ Turkish Journal of Urology. 45(3):164-170

\section{Publisher's Note}

Springer Nature remains neutral with regard to jurisdictional claims in published maps and institutional affiliations.

\section{Submit your manuscript to a SpringerOpen ${ }^{\circ}$ journal and benefit from:}

- Convenient online submission

- Rigorous peer review

- Open access: articles freely available online

- High visibility within the field

- Retaining the copyright to your article

Submit your next manuscript at $\boldsymbol{\nabla}$ springeropen.com 